\title{
GaN-HEMTs Cascode Switch: Fabrication and Demonstration on Power Conditioning Applications
}

\author{
Stone Cheng*, Po-Chien Chou \\ Dept. of Mechanical Eng., National Chiao-Tung University, 1001 University Road, Hsinchu, Taiwan 300, ROC \\ *Corresponding Author: stonecheng@mail.nctu.edu.tw
}

\begin{abstract}
A novel metal TO-257 4-pin package is proposed for the packaging of a D-mode GaN MIS-HEMTs cascoded with an integrated power MOSFET and a Schottky diode device, and demonstrated in power conversion applications. The normally-off hybrid cascode circuit provides a maximum drain current of $14.6 \mathrm{~A}$ and a blocking capability of $600 \mathrm{~V}$. The cascode configuration has a $+3.9 \mathrm{~V}$ gate threshold, sufficient voltage swing, good controllability of commonly available gate drivers. Analysis of $48 \mathrm{~V} / 2$ A power conversion characteristics are discussed and show the excellent switching performance in inductive load circuits. Switching characteristics of the integral SiC SBD are also demonstrated. Finally, a 48-to-96 V boost converter is used to evaluate the benefit of $\mathrm{GaN}$ cascode switches. Experimental results show an output power of $94.8 \mathrm{~W}$ and a power efficiency of $95.37 \%$ under a switching frequency of $100 \mathrm{kHz}$, which is higher than the $92.03 \%$ power efficiency provided by the conventional Si MOSFET with the same switching operation.
\end{abstract}

Keywords: GaN-HEMT cascode switch, DC-DC conversion, power semiconductor device.

\section{Introduction}

Due to the extraordinary material properties of GaN, GaN HEMTs have been used in many power conversion and RF applications ${ }^{(1,2)}$. Aggressive development of GaN in large-diameter (up to $200 \mathrm{~mm}$ ) Si wafers combined with the development CMOS compatible processes has resulted in a cost effective, high performance technology in comparison with SiC-based technology ${ }^{(3,4)}$. The GaN high electron mobility transistors (HEMTs) are now used in micro-inverters $^{(5,6)}$. For power switching applications, the enhancement mode (E-mode) devices feature a narrow gate voltage swing, which requires highly accurate control to prevent spikes ${ }^{(7,8)}$. For safety reasons, depletion mode (D-mode) devices require a negative drive voltage, which is difficult to provide with commonly available gate drivers $^{(9,10)}$. To avoid the deficiencies of the above approaches, this study combines a high voltage (HV) D-mode GaN HEMT device with a low voltage (LV) Si-based MOSFET device in cascode configuration ${ }^{(11-13)}$. Depletion mode devices in a cascade structure behave like MOS transistors but with high voltage and low on-resistance characteristics. This study presents the optimal cascode configuration in terms of device physics, process technology, design and advanced packaging, and performance. A D-mode GaN device with a metal-insulator-semiconductor (MIS) gate structure has been developed for high-voltage power switches ${ }^{(14,15)}$. The advantage of the D-mode MIS-HEMT compared to its conventional equivalent is its enlarged gate swing with a lower gate leakage current. For a given power rating, $\mathrm{GaN}$ devices provide 10 times higher power density compared to Si-based devices ${ }^{(16)}$. However, although various studies have considered the on-wafer power characteristics of discrete GaN HEMTs at the load-pull level, none has considered the effects of chip-size packages. Due to the very small die surface area, proper thermal management is critical $^{(17,18)}$. Details of the materials and processes used to fabricate the package are discussed. This study analyzed the performance characteristics of a D-mode MIS-HEMT device configured in cascode mode, including the switching characteristics of the device and its integration into a hermetically sealed 4-pin TO-257 metallic package ${ }^{(19,20)}$. A fourth pin is added to provide a Kelvin source and to reduce the parasitic inductance of the source lead of the power MIS-HEMT. An added benefit of the Kelvin source 
configuration in the TO 257-4 is the ease of using the PCB layout due to its new pin arrangement, in which the drain is exposed and the source is between the gate and drain. This reduces the critical drain to gate coupling, which often causes gate oscillations at turn off when the Drain-Source Voltage $\left(\mathrm{V}_{\mathrm{DS}}\right)$ has high $\mathrm{dv} / \mathrm{dt}$ rates. This in-package hybrid cascade circuit with an integrated SiC Schottky barrier diode (SBD) effectively bypasses the slow and inefficient internal body diode of the Si MOSFET ${ }^{(21)}$. The relevance of GaN cascode switches to power electronics was discussed $^{(22,23)}$. Current switching speeds of GaN cascode circuits are higher than those of Si MOSFETs in the same voltage range. In the analysis of converter design and loss, clamped inductive load switching measurements are performed to evaluate switching performance and to provide loss references ${ }^{(24)}$. Switching characteristics of the integral SiC SBD are also demonstrated. Finally, A boost converter is used in further performance tests to evaluate the feasibility and advantages of $\mathrm{GaN}$ cascode switches in power applications.

\section{AlGaN/GaN HEMTS Device Structure and Fabrication}

Recent technological improvements in $\mathrm{GaN}$ on silicon have enabled large-area GaN HEMT power devices. The largest single chip device fabricated on a GaN-on-Si wafer to date is an $80 \mathrm{~A} / 600 \mathrm{~V}$ GaN-based power device with an active area of $8 \mathrm{~mm} 2$. The availability of large-area devices does not mean they are economically viable in large-volume production. As in silicon, the die cost increases dramatically as the device size increases because the growth uniformity and processing yield decrease. These limitations are also the main reason why $\mathrm{SiC}$ power modules are fabricated by bonding multiple lower current die in parallel until a desired high current rating. Figure 1 shows the D-mode AlGaN/GaN MIS-HEMT structure studied in this work. The device had 80 gate fingers, a gate length of $1 \mu \mathrm{m}$, a gate width of $1 \mathrm{~mm}$, and a total gate periphery of $80 \mathrm{~mm}$. Deposition and fabrication were performed on 4-inch $\mathrm{Si}$ substrates to determine process uniformity. The AlGaN/GaN hetero-structure pattern is grown on a silicon substrate (thickness, $900 \mu \mathrm{m}$ ) by metalorganic chemical vapor deposition. The epitaxial structure consisted of a $3.9 \mu \mathrm{m}$-thick GaN/AlGaN buffer layer, on which was deposited a $2.1 \mu \mathrm{m}$-thick GaN layer, a 20 nm-thick $\mathrm{Al}_{0.23} \mathrm{Ga}_{0.77} \mathrm{~N}$ barrier layer and, finally, a 4 nm-thick GaN capping layer. To fabricate this device, Mesa isolation was performed with a ICP-RIE system with $\mathrm{Cl}_{2}$ as the etching gas. Source/drain Ohmic contacts were formed by an alloyed Ti/Al/Ni/Au (20 nm / 120nm / 25nm / $100 \mathrm{~nm}$ ) metal stack. The $2 \mathrm{~nm}$ AlN interfacial passivation layer (IPL) was deposited by PE-ALD system with $\mathrm{Al}\left(\mathrm{CH}_{3}\right)_{3}$ (TMA) and $\mathrm{NH}_{3}$ gases as precursors. The same chamber was then used to deposit $8 \mathrm{~nm} \mathrm{Al}_{2} \mathrm{O}_{3}$. Afterward, post deposition annealing (PDA) was performed at $500{ }^{\circ} \mathrm{C}$ in N2 ambient to improve the gate insulator quality. The $2 \mathrm{~nm}$ AlN IPL has proven effective for suppressing threshold voltage hysteresis and for reducing current collapse $^{(25)}$. The Ni/Au (50nm / 450nm) was used as the gate electrode on top of the dielectric stack. Finally, the device was covered with a 200 nm-thick $\mathrm{Si}_{3} \mathrm{~N}_{4}$ film for device passivation and provided with a standard Au-plated interconnect to complete a multi-finger FET.

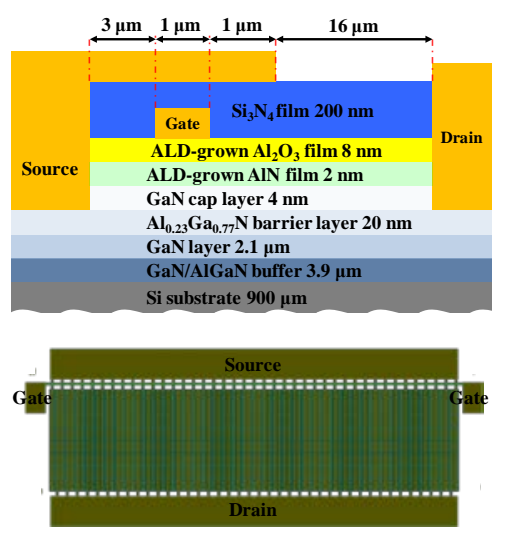

Fig. 1. Cross sections of fabricated MIS-HEMT structure for depletion-mode operation. The gate periphery is $80 \mathrm{~mm}$ wide (gate length, $1 \mu \mathrm{m}$; gate width, $1 \mathrm{~mm}$ ).

For maximum acceptability and applicability, the hybrid cascade circuit allows D-mode MIS-HEMTs to function like E-mode devices as shown in Fig. 2(a). This GaN power switch incorporates a low-voltage (LV) MOSFET connected in series with the high-voltage (HV) GaN HEMTs, wherein the internal SiC SBD connects anti-parallel to the cascode. The diode co-packaged with the cascode structure reduces power consumption and enables support for smaller peripheral components. Using the on-off state of an LV MOSFET to control the on-off state of D-mode MIS-HEMTs makes the cascode power switch compatible with existing Si gate-drivers. The $\mathrm{HV} \mathrm{GaN}$ HEMT extends the operating voltages of power systems, and the LV MOSFET provides a sufficient safety driving margin. This configuration helps to mitigate gate drive issues because E-mode HEMTs require a drive of $5 \mathrm{~V}$ to become fully enhanced but suffer damage if the gate drive 
rises above $6 \mathrm{~V}$. The design and implementation of the cascode configured GaN Switch was also considered. Figure 2(b) shows the proposed cascode-connected GaN HEMTs in a TO-257 style metal package ${ }^{(26,27)}$. A fourth pin is added to provide a Kelvin source connection (SK), which is used only for switching control. This connection reduces the common source inductance of the main power connection. The HV and LV devices are bonded on a direct bond copper (DBC) AlN substrate in a metal-encapsulated package. The DBC substrates are patterned to establish the required interconnects between the devices. The design space of the substrate layout was fully searched to enable reduced parasitic parameters and layout area. Both devices were attached to the DBC substrate by silver sintering, and the DBC substrate was attached to the metal header by vacuum vapour phase soldering. The gate, drain and source were connected to package terminals by $0.03 \mathrm{~mm}$ diameter gold wire bonds. The module was then filled with silicon gel to ensure good thermal stability and electrical insulation. Finally, a coefficient of expansion (CTE) matched metal lid was sealed in place.
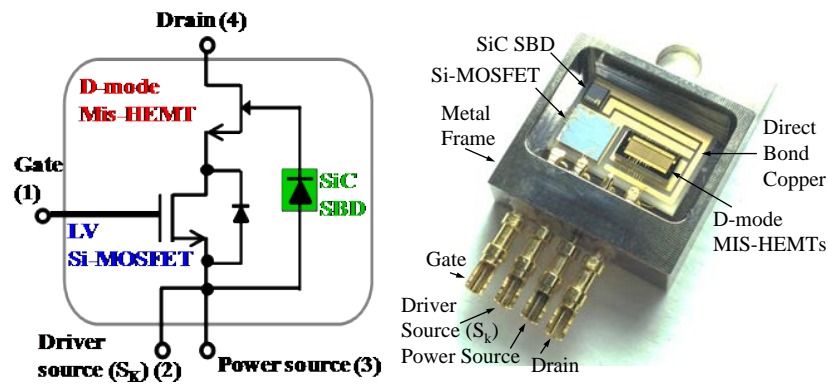

Fig. 2. Cascode GaN HEMT structure. (a) A schematic diagram of the cascode circuit showing the normally-on MIS-HEMT, the normally-off LV Si-MOSFET, and the SiC SBD control circuitry. (b) Photograph of prototype TO-257 4-pin metallic package with Kelvin source connection (SK).

\section{Characterization of GaN-Cascode Device}

Many studies have evaluated the performance evaluations of cascoded GaN HEMT devices. The cascode switch is mainly tested in terms of output characteristics $\left(\mathrm{ID}-\mathrm{V}_{\mathrm{DS}}\right)$, transfer characteristics $\left(\mathrm{ID}-\mathrm{V}_{\mathrm{GS}}\right)$ and voltage blocking capability. For the completed device, Fig. 3(a) shows the output characteristics obtained by a Keithley $2651 \mathrm{~A}$ source meter with proper biasing where the $V_{\mathrm{GS}}$ starts from $0 \mathrm{~V}$ to $8 \mathrm{~V}$ in steps of $2 \mathrm{~V}$. When $\mathrm{V}_{\mathrm{GS}}=2 \mathrm{~V}$, the drain current is almost zero; when $\mathrm{V}_{\mathrm{GS}}=6 \mathrm{~V}$, the combined switch is fully on and carries a drain current in excess of 14.6 A with a voltage drop of $5 \mathrm{~V}$. When connected in series, the $30 \mathrm{~V}$ MOSFET (Ron $30 \mathrm{~m} \Omega$ ) increases the total cascode on-resistance by $330 \mathrm{~m} \Omega$, which is less than $10 \%$. Most conduction loss occurs in the HV GaN HEMTs. Figure 3(b) shows the normally-off transfer characteristics (ID- $\mathrm{V}_{\mathrm{GS}}$ ) for $\mathrm{V}_{\mathrm{GS}}$ from $0-6 \mathrm{~V}$ in $0.1 \mathrm{~V}$ increments and $\mathrm{V}_{\mathrm{DS}}$ ranging from 1 - $5 \mathrm{~V}$. A gate-threshold voltage higher than $+3.5 \mathrm{~V}$ provides a sufficient margin for safe operation. Since the cascode switch can sustain a large input-gate voltage swing $(>5 \mathrm{~V}$ ) without failure, D-mode HEMTs are compatible with existing gate drive circuits. In contrast, the gate voltage swing of a commercially available E-mode HEMT is $1-1.5 \mathrm{~V}$.

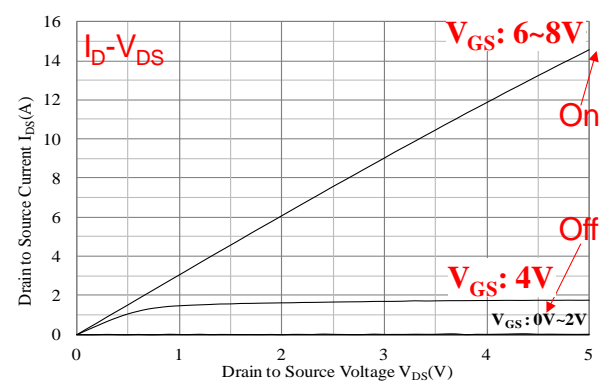

(a)

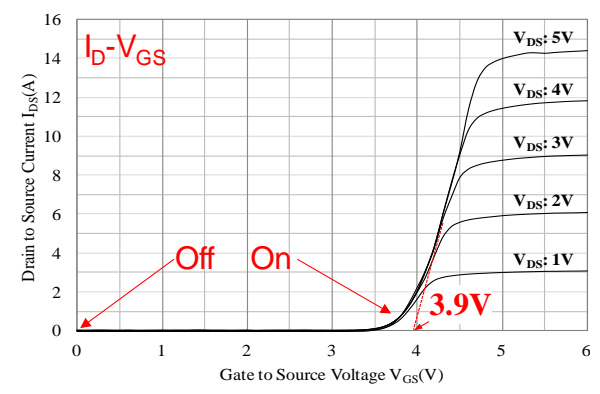

(b)

Fig. 3. (a) Measured output characteristics (ID- $\mathrm{V}_{\mathrm{DS}}$ ) of a GaN-based cascode switch with an active area of $4 \mathrm{~mm} 2$ and a total gate periphery of $80 \mathrm{~mm}$ at $25^{\circ} \mathrm{C}$. (b) Measured Transfer characteristics (ID- $\mathrm{V}_{\mathrm{GS}}$ ) of the cascode switch in a TO-257 package at $25^{\circ} \mathrm{C}$.

The GaN power switch shows high voltage blocking capability at room temperature $\left(25^{\circ} \mathrm{C}\right)$. Figure 4 displays the off-state characteristics of the cascode switch. As $V_{D S}$ is swept forward from $0 \mathrm{~V}$ to $650 \mathrm{~V}$, the maximum voltage at which leakage is limited to $10 \mathrm{~mA}$ increased steadily. The LV MOSFET (leakage current $25 \mu \mathrm{A} @$ max) defines and controls the on/off state of HV GaN HEMTs, which results in an off-state for $\mathrm{V}_{\mathrm{GS}}=0 \mathrm{~V}$. The high voltage off-state characteristics reveal that the leakage current (approximately $5.8 \mathrm{~mA}$ at $600 \mathrm{~V}$ ) is mainly from D-mode MIS-HEMTs and is four orders of magnitude lower than the maximum on-state current. The resulting leakage current is 
lower than $100 \mu \mathrm{A} / \mathrm{mm}$ of gate width. Off-state breakdown characteristics were further analyzed in the D-mode HEMTs with and without additional AlN/Al2O3 bi-layer gate dielectric. Figure 5 shows that the drain leakage current is measured at a $V_{G S}$ value of $-4 \mathrm{~V}$ while increasing $\mathrm{V}_{\mathrm{DS}}$ to $650 \mathrm{~V}$. The breakdown voltage of MIS-HEMT with bi-layer gate dielectric $(620 \mathrm{~V})$ is significantly higher than that of conventional HEMT (340 V). Notably, dislocations and other defects in the conventional HEMT result in a high drain leakage current. These experimental results suggest that, when used in cascode configuration, MIS-HEMTs effectively suppress forward leakage current and current collapse under high drain bias switching conditions. The HEMT leakage current must be sufficiently low to reduce power loss in the cascode switch.

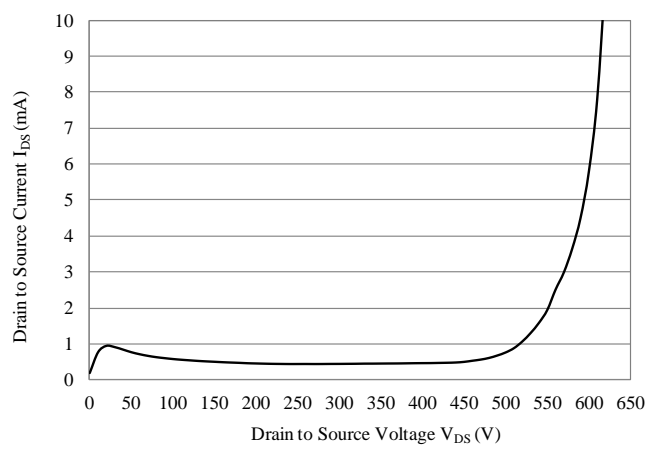

Fig. 4. Drain leakage currents of cascoded GaN switch as a function of drain bias wth $\mathrm{V}_{\mathrm{GS}}$ fixed at $0 \mathrm{~V}$.

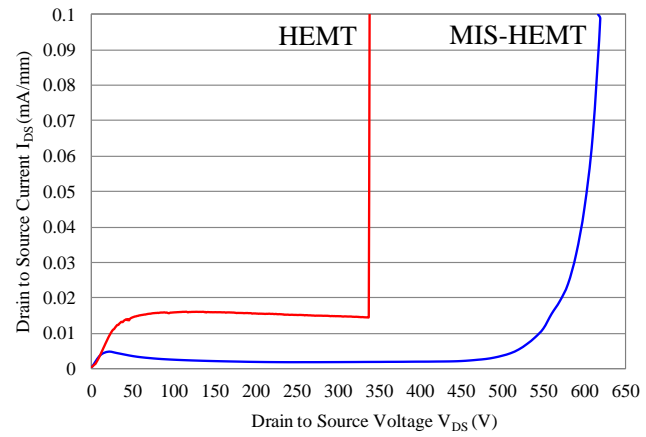

Fig. 5. Drain leakage current of $80 \mathrm{~mm}$ gate periphery D-mode HEMTs with and without additional bi-layer gate dielectric. The $\mathrm{V}_{\mathrm{GS}}$ is fixed at $-4 \mathrm{~V}$.

\section{Switching Characterization}

Figure 6 represents the $48 \mathrm{~V} / 2$ A clamped inductive load characterization test circuit which evaluates the switching performance applied to the DUT in power conversion applications. In this circuit, a low inductive path is provided for the transient current during switching to eliminate parasitic oscillations where possible. The cascade GaN HEMT package is gated on using a double pulse gate voltage. The first pulse $(19.8 \mu \mathrm{s})$ builds up the test current in the inductor. When the first pulse is terminated, the inductor current commutates from the DUT to the adjacent SiC SBD. This short turn-off period is used to measure the DUT turn-off switching characteristics. The next adjacent pulse $(2.5 \mu \mathrm{s})$ occurs $5 \mu$ s later. Upon turn-on of the DUT gate during this second pulse, the test current flowing into the inductor current combined with the diode reverse current creates a current spike in the DUT. The inductive load creates a situation where high-voltage and high-current are simultaneously applied to the DUT. Figure 7 shows the switch waveforms for a 10-ohm gate resistor connected in series in the gate biasing circuit. Because of the fast switching transients, both the switched current and voltage waveforms have large ringing during the turn on. The switch waveforms are mainly to provide the loss reference to the design of the boost converter in the following sections

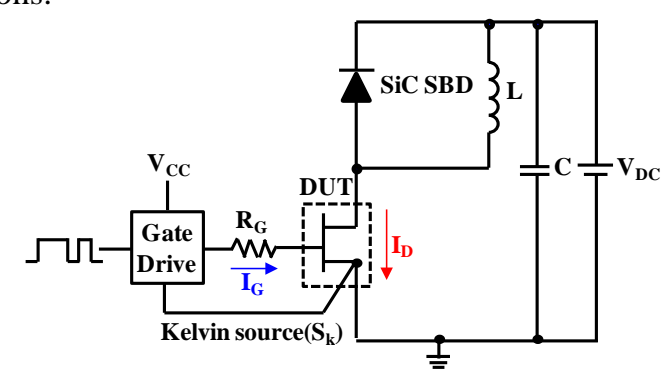

Fig. 6. Clamped inductive load test circuit used to emulate application conditions for analysis of double pulse switching.

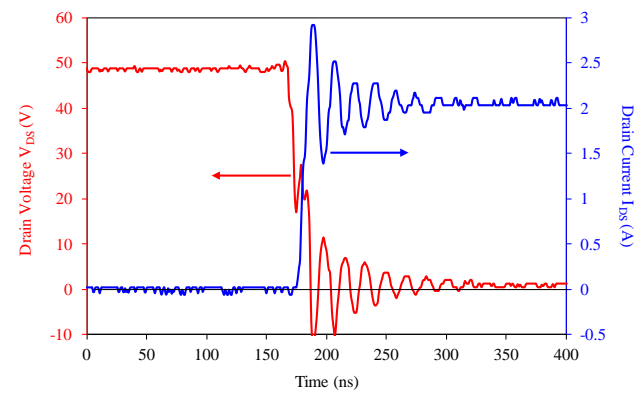

(a)

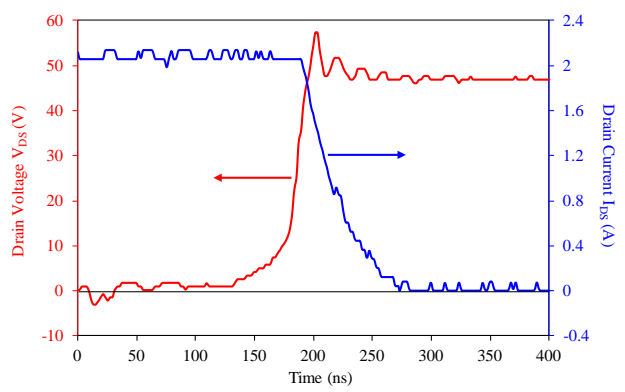

(b)

Fig. 7. Clamped inductive load circuit for double pulse switching waveforms at (a) turn-on and (b) turn-off. 
Figure 8(a) shows the test system used to characterize the reverse recovery characteristics of integrated diodes. The DUT gate and source are shorted to test the cascode configuration with and without an integrated SiC SBD. The other GaN switch acts as a control device and is subjected to a double pulse. The first pulse is used to establish the test current in the inductor (L). The current ramps in the lower control device and freewheels through the DUT internal diode during turn-off of the control device. When it is turned on again by the second pulse, the DUT internal diode begins to recover the DUT voltage rises toward the bus voltage VDC, which completes the recovery test. The cascode GaN switch exhibits outstanding reverse recovery characteristics. Figure 8(b) shows the reverse recovery characteristics of cascoded switches with (solid line) and without (dashed line) an integrated SiC SBD. The reverse recovery time trr for cascoded switches with SiC SBD is short (less than 1/2), and the reverse recovery charge Qrr is small (less than 1/5) as compared with those without $\mathrm{SiC}$ SBD. The implications of a low Qrr are the potential to achieve a lower conduction and switching losses in the boost converter. A low Qrr also minimizes the ringing and overshoot voltage to ensure sufficient reliable operation.

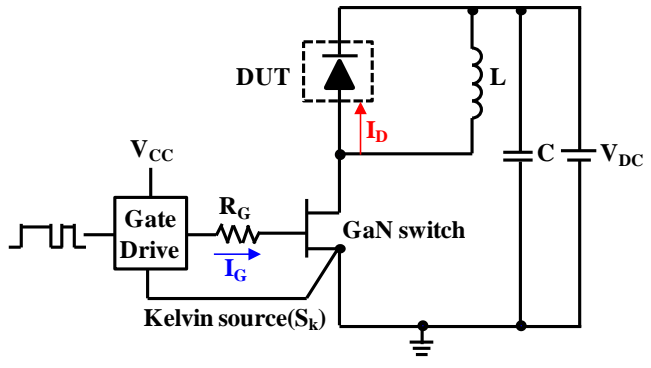

(a)

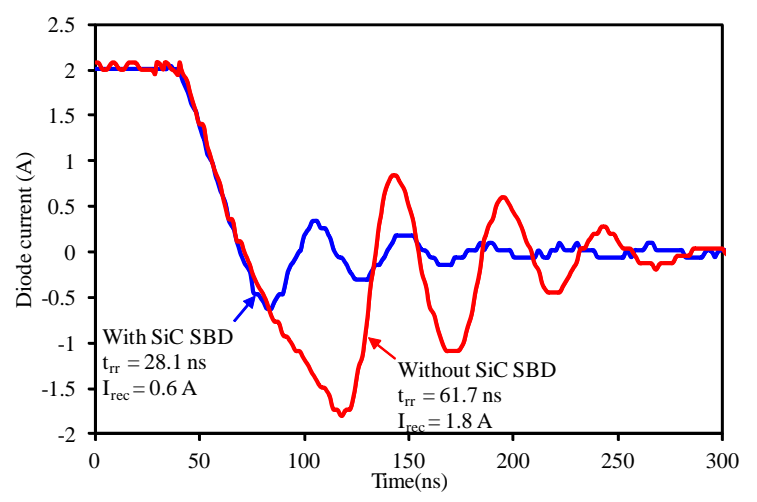

(b)

Fig. 8. (a) Diode reverse recovery test circuit. (b) Reverse recovery characteristics for the $48 \mathrm{~V} / 2$ A GaN cascode circuit with and without an integrated SiC SBD.

A 96 W DC-DC boost converter prototype is built with single GaN cascade switch and SiC SBD. Figure 9(a) shows a diagram of the studied circuit. The input and output voltages are $48 \mathrm{~V}$ and $96 \mathrm{~V}$, respectively. The main circuit consists of an inductor (L), a GaN cascode switch, a SiC SBD, two filtration capacitors (Cin and Cout), and a load (Rload). The SiC SBD is used in the experiment due to their superior switching characteristics with zero recovery current ${ }^{(28)}$. The inductor absorbs energy during the on-interval of the GaN cascade switch and releases the stored energy to the load at twice the input voltage during the off-interval of the switch. A gate-source voltage $\left(\mathrm{V}_{\mathrm{GS}}\right)$ swing from 0 to $12 \mathrm{~V}$ is supplied using a pulse generator with $50 \%$ duty cycle and $100 \mathrm{kHz}$ frequency. The boost converter operates in continuous-conduction mode (CCM) to avoid inductor current becoming discontinuous. Circuit performance was evaluated under actual operating conditions by building the power circuit on a dual-layer PCB with the backside soldered on the copper heat sink. The back of the HEMT and SBD tabs were bolted onto the heat spreader to provide grounding and additional heat transfer paths. Since the MIS-HEMT structure minimized the impacts of the forward drain bias stress, Fig. 9(b) shows that the circuit provided a continuous stable operation of 96 $\mathrm{V}$ and maintained high power efficiency. Power efficiency was $95.37 \%$, and output power was $94.8 \mathrm{~W}$. Leakage currents from the LV MOSFET and HV HEMT cause further losses; therefore, the leakage current levels of the MIS-HEMTs are maintained below $1 \mathrm{nA} / \mathrm{mm}$. Therefore, they do not affect the overall efficiency of the overall cascode configuration $^{(29)}$. This output power is the same level as that for the power supply and battery charger applications. Figure 10 shows the efficiency curves of Si-based and GaN-based boost converter. In this evaluation, all the DUTs are placed in a test fixture under the same operating conditions except that the GaN cascade switch is replaced with the power MOSFET while the SiC SBD is still used as the boost diode. The performance is compared in terms of the measured converter efficiencies between a HEXFET ${ }^{\circledR}$ MOSFET (International Rectifier, part IRF540ZPbF) and a GaN cascade switch ${ }^{(30)}$. The efficiency of the GaN-based converter improves by $3.3 \%$, mainly through the improved switching loss. The efficiency difference exceeds $3.8 \%$ at $17 \mathrm{~W}$ output power under a light load because the conduction loss becomes a small percentage of the total converter loss. The analyses of reverse recovery characteristics show even more advantages of the hybrid cascode circuitry. 


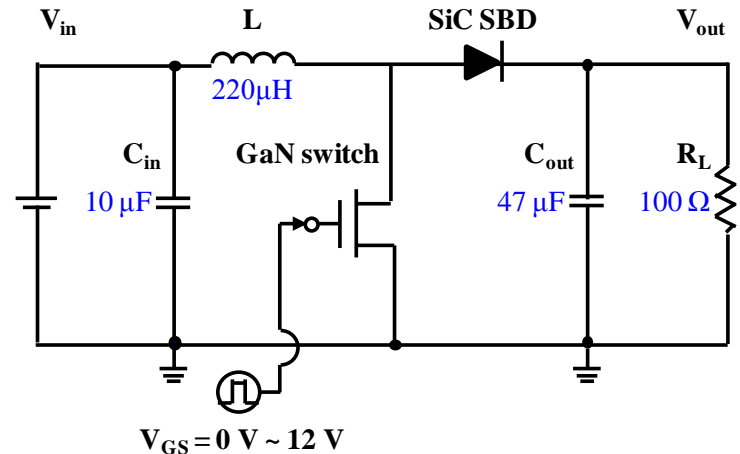

(a)

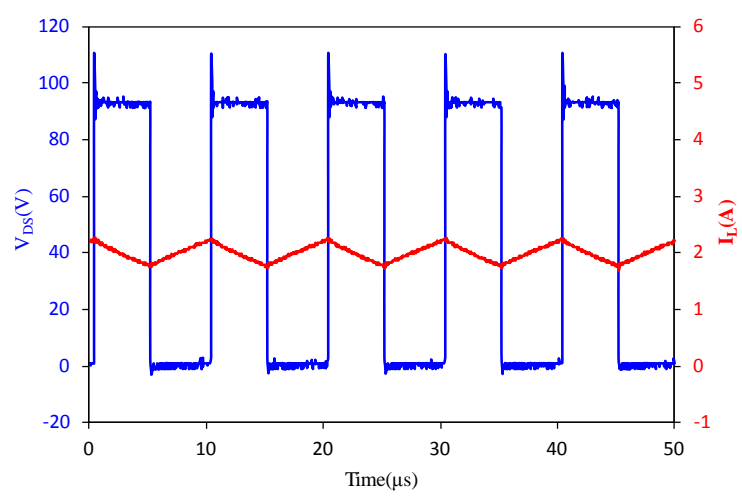

(b)

Fig. 9. Evaluated boost converter circuit with cascoded GaN switch as a main switching-device. (a) Circuit diagram of the boost converter. (b) Voltage and current waveforms measured at the drain of the GaN switch and through the inductor (L), respectively. The operating parameters included a duty cycle of $50 \%$, and a dc input and output of $48 \mathrm{~V}$ at $2.07 \mathrm{~A}$ and $96.7 \mathrm{~V}$ at $0.98 \mathrm{~A}$, respectively.

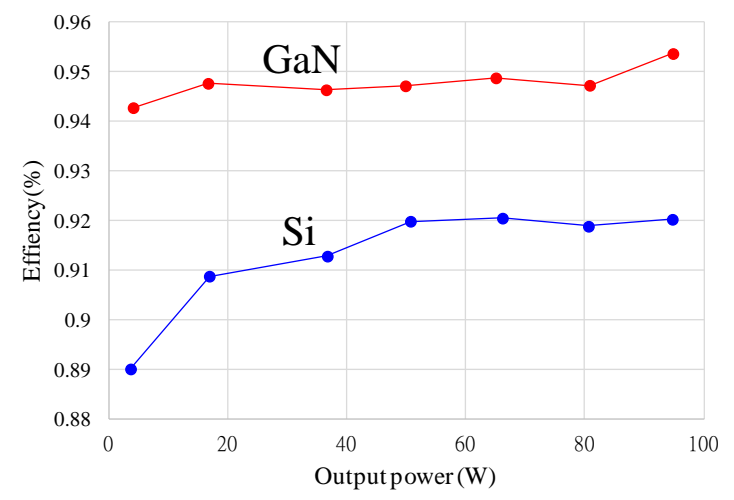

Fig. 10. Efficiencies of Si-based and GaN-based boost converter.

\section{Conclusions}

This study successfully demonstrated the integration of a D-mode GaN MIS-HEMT with a Si MOSFET and SiC SBD in a cascode configuration. The metal TO-257 4-pin package developed for a cascode structure provided sufficient local thermal management to dissipate heat, which is mainly produced by the GaN HEMTs. A fourth pin added to provide a Kelvin source connection for gate-driven control effectively reduces drain-gate coupling, which causes gate oscillations. The proposed normally-off hybrid cascode circuit has a maximum drain current of 14.6 A and a breakdown voltage exceeding $600 \mathrm{~V}$. The cascode configuration, which has a $+3.9 \mathrm{~V}$ gate threshold and an enlarged gate voltage swing, is easily driven by commonly available gate drivers. Standard double-pulse clamped inductive load test is performed to demonstrate the durability of the cascode. Reverse recovery characteristics of the cascade circuit are demonstrated with and without internal SiC SBD. Finally, a 48-to-96 V boost converter is explored to evaluate the benefit of hybrid cascode switches. Experimental results show power efficiency of $95.37 \%$ under a switching frequency of $100 \mathrm{kHz}$, which is higher than that the $92.03 \%$ power efficiency of a Si MOSFET in the same switching operation.

\section{Acknowledgment}

This work was supported by the MOEA project 101-EC-17-A-05-S1-154, and MOST project 102-2221-E-009-074, Taiwan, R.O.C..

\section{References}

(1) U. K. Mishra, P. Parikh and Y. F. Wu, “AlGaN/GaN HEMTs-an overview of device operation and application”, Proc. IEEE,90 (2002) 1022-31.

(2) U. K. Mishra, L. Shen, T. E. Kazior, and Y. F. Wu, “GaN-based RF power devices and amplifiers”, Proc. IEEE, vol. 96 (2) (2008) pp. 287-305.

(3) W. E. Hoke, T. D. Kennedy, A. Torabi, P. S. Lyman, C. A. Howsare, and B. D. Schultz, "Highly uniform AlGaN/GaN HEMT films grown on 200-mm silicon substrates by plasma molecular beam epitaxy”, J. Vac. Sci. Technol. B, vol. 32 (2) (2014) 030605.

(4) D. Marcon, B. De Jaeger, S. Halder, N. Vranckx, G. Mannaert, M. Van Hove and S. Decoutere "Manufacturing challenges of GaN-on-Si HEMTs in a 200 mm CMOS fab”, IEEE Trans. Semicond. Manuf., vol. 26, (3) (2013) pp.361-367.

(5) A. Lidow, J. Strydom, M. de Rooij and Y. Ma "GaN Transistors for Efficient Power Conversion”, (2012) Power Conversion Publications.

(6) L. A. Garcia Rodrigues, E. Williams, J.C. Balda, and C. Busada, "Dual-Stage Microinverter Design of a GaN-based Interleaved Flyback Converter Stage”, IEEE ECCE (2013) pp. 4496-4502. 
(7) J. Delaine, P. Olivier, D. Frey, and K. Guepratte, "High frequency DC-DC converter using GaN device”, IEEE APEC Dig. (2012) pp.1754-1761.

(8) D. Reusch, F. C. Lee, D. Gilham, and Y. Su, "Optimization of a high density gallium nitride based non-isolated point of load module”, IEEE ECCE (2012) pp.2914-2920.

(9) G. Kampitsis, S. Papathanassiou, and S. Manias, "Performance consideration of an AC coupled gate drive circuit with forward bias for normally-on SiC JFETs", IEEE ECCE (2013) pp. 3224-3229.

(10)B. Wrzecionko, D. Bortis, J. Biela and J. W. Kolar, "Novel AC-coupled gate driver for ultrafast switching of normally off SiC JFETs", IEEE Trans. Power Electron., vol. 27, (7) (2012) pp.3452-3463.

(11)X. Huang, Z. Liu, Q. Li and F. C. Lee, "Evaluation and application of $600 \mathrm{~V}$ GaN HEMT in cascode structure", Proc. Twenty-Eighth Annu. IEEE Appl. Power Electron. Conf. Expo., (2013) pp.1279-1286.

(12)X. Huang, Q. Li, Z. Liu, and F. C. Lee, "Analytical loss model of high voltage GaN HEMT in cascode configuration”, IEEE Trans. Power Electron., vol. 29, (5) (2014) pp. 2208-2219.

(13) W. Zhang, Z. Xu, Z. Zhang, F. Wang, L. M. Tolbert, and B. J. Blalock, "Evaluation of $600 \mathrm{~V}$ cascode GaN HEMT in device characterization and all-GaN-based LLC resonant converter", IEEE ECCE (2013) pp. 3571-3578.

(14) T. Wu, D. Marcon, M. Zahid, M. Van Hove, S. Decoutere, and G. Groeseneken "Comprehensive investigation of on-state stress on d-mode AlGaN/GaN MIS-HEMTs", Proc. IEEE Int. Rel. Phys. Symp., (2013) pp.3C.5.1 -3C.5.7.

(15) M. Meneghini et al., "Trapping and reliability assessment in D-mode GaN-based MIS-HEMTs for power applications”, IEEE Trans. Power Electron., vol. 29 (5) (2014) pp. 2199-2207.

(16) U. K. Mishra, P. Parikh and Y.-F. Wu, "AlGaN/GaN HEMTs-An overview of device operations and applications", Proceedings of the IEEE, vol. 90, (6) (2002) pp.1022-1031.

(17)A. Bar-Cohen, J. D. Albrecht, and J. J. Maurer,, "Near Junction Thermal Management for Wide Bandgap Devices," IEEE Symposium on Compound Semiconductor Integrated Circuit, (2011) pp. 1-5.

(18) Y. Won, J. Cho, D. Agonafer, M. Asheghi, and K. E. Goodson, "Cooling Limits for GaN HEMT Technology”, IEEE Symposium on Compound
Semiconductor Integrated Circuit, (2013) pp. 1-5.

(19) Z. Liu, X. Huang, W. Zhang, F. C. Lee, and Q. Li "Evaluation of High-Voltage Cascode GaN HEMT in Different Packages”, IEEE APEC Dig. (2014) pp.168-173.

(20) S. Cheng, P.C. Chou, W.H. Chieng, and E.Y. Chang, "Enhanced lateral heat dissipation packaging structure for GaN HEMTs on Si substrate”, Appl. Therm. Eng. 51 (1-2) (2013) 20-24.

(21) J. J. Zhang "GaN-based device cascoded with an integrated FET/Schottky diode device”, U.S. Patent Application Publication, US 8084783 B2 (2011)

(22) T. McNutt et al, "Silicon carbide JFET cascode switch for power conditioning applications", IEEE VPPC (2005) pp 217-226.

(23) N. Zhang, V. Mehrotra, S. Chandrasekaran, B. Moran, L. Shen, U. Mishra, E. Etzkorn and D. Clarke, "Large area GaN HEMT power devices for power electronic applications: Switching and temperature characteristics”, IEEE PESC (2003) pp.233-237.

(24) Y. Wu, M. Jacob-Mitos, M. L. Moore and S. Heikman, "A 97.8\% efficient GaN HEMT boost converter with 300-W output power at $1 \mathrm{MHz}$, IEEE Electron Device Lett., vol. 29 (8) (2008) pp.824 -826.

(25)T. E. Hsieh, Chang, E.Y., Y.Z. Song; Y.C. Lin; H. C. Wang; S. C. Liu; Salahuddin, S.; Hu, C.C., "Gate Recessed Quasi-normally OFF Al2O3/AlGaN/GaN MIS-HEMT with Low Threshold Voltage Hysteresis using PEALD AlN Interfacial Passivation Layer", IEEE Elec. Dev. Lett., vol. 35 (7) (2014) pp.732 -734.

(26) P.C. Chou, S. Cheng, Design and characterization of a 200 V, 45 A all-GaN HEMT-based power module, Appl. Therm. Eng. 61 (2013) 20e27.

(27) S. Cheng a, P.C. Chou, W.H. Chieng, E.Y. Chang, Enhanced lateral heat dissipation packaging structure for GaN HEMTs on Si substrate, Appl. Therm. Eng. 51 (2013) 20e24.

(28) J. Everts, J. Das, J. Van den Keybus, J. Genoe, Germain, Marianne, and J. Driesen, “A High-Efficiency, High-Frequency Boost Converter using Enhancement Mode GaN DHFETs on Silicon”, IEEE ECCE (2010) pp. 3296-3302.

(29) P. Parikh, Y. Wu, L. Shen, "Commercialization of high 600V GaN-on-silicon power HEMTs and diodes”, Energytech, 2013 IEEE. IEEE, 2013.

(30) Datasheet-International Rectifier http://www.irf.com/product-info/datasheets/data/irf540 zpbf.pdf 